TRANSACTIONS OF THE

AMERICAN MATHEMATICAL SOCIETY

Volume 361, Number 10, October 2009, Pages 5401-5416

S 0002-9947(09)04790-4

Article electronically published on May 11, 2009

\title{
INSTABILITY FOR STANDING WAVES OF NONLINEAR KLEIN-GORDON EQUATIONS VIA MOUNTAIN-PASS ARGUMENTS
}

\author{
LOUIS JEANJEAN AND STEFAN LE COZ
}

\begin{abstract}
We introduce mountain-pass type arguments in the context of orbital instability for Klein-Gordon equations. Our aim is to illustrate on two examples how these arguments can be useful to simplify proofs and derive new results of orbital stability/instability. For a power-type nonlinearity, we prove that the ground states of the associated stationary equation are minimizers of the functional action on a wide variety of constraints. For a general nonlinearity, we extend to the dimension $N=2$ the classical instability result for stationary solutions of nonlinear Klein-Gordon equations proved in 1985 by Shatah in dimension $N \geqslant 3$.
\end{abstract}

\section{INTRODUCTION}

The aim of the present paper is to show how recent methods and results concerning the variational characterizations of the ground states for elliptic equations of the form

$$
-\Delta \varphi=g(\varphi), \quad \varphi \in H^{1}\left(\mathbb{R}^{N} ; \mathbb{C}\right)
$$

can be used to study the orbital stability/instability of the standing waves of various nonlinear equations such as Schrödinger equations, Klein-Gordon equations, generalized Boussinesq equations, etc. Our work is motivated by recent developments (see, for instance, [9, 16, 17, 18, 22, 23) of the techniques introduced by Berestycki and Cazenave [2] to prove the instability of standing waves for nonlinear evolution equations. We present our approach on two examples involving nonlinear Klein-Gordon equations of the form

$$
u_{t t}-\Delta u+\rho u=f(u),
$$

where $\rho>0, u: \mathbb{R} \times \mathbb{R}^{N} \mapsto \mathbb{C}$ and $f:(0,+\infty) \mapsto \mathbb{R}$ is extended to $\mathbb{C}$ by setting $f(z)=f(|z|) z /|z|$ for $z \in \mathbb{C} \backslash\{0\}$ and $f(0)=0$.

A standing wave of (2) is a solution of the form $e^{i \omega t} \varphi_{\omega}(x)$ for $\omega \in \mathbb{R}$ and $\varphi_{\omega} \in$ $H^{1}\left(\mathbb{R}^{N} ; \mathbb{C}\right)$. Thus $\varphi_{\omega}$ satisfies

$$
-\Delta \varphi_{\omega}+\left(\rho-\omega^{2}\right) \varphi_{\omega}-f\left(\varphi_{\omega}\right)=0 .
$$

Clearly, (3) is of the form (11). From now on we write $H^{1}\left(\mathbb{R}^{N}\right)$ for $H^{1}\left(\mathbb{R}^{N} ; \mathbb{C}\right)$. The least energy level $m$ is defined by

$$
m:=\inf \left\{S(v) \mid v \in H^{1}\left(\mathbb{R}^{N}\right) \backslash\{0\}, v \text { is a solution of (10) }\right\},
$$

Received by the editors October 16, 2007.

2000 Mathematics Subject Classification. Primary 35Q53, 35B35, 35A15, 35Q51.

(C)2009 American Mathematical Society 
where $S: H^{1}\left(\mathbb{R}^{N}\right) \mapsto \mathbb{R}$ is the natural functional (often called action) corresponding to (11),

$$
S(v):=\frac{1}{2}\|\nabla v\|_{2}^{2}-\int_{\mathbb{R}^{N}} G(v) d x,
$$

with $G(s):=\int_{0}^{|s|} g(t) d t$. A solution $\varphi \in H^{1}\left(\mathbb{R}^{N}\right)$ of (1) is said to be a ground state, or least energy solution, if

$$
S(\varphi)=m .
$$

The study of the existence for solutions of (11) goes back to the work of Strauss [26] (see also [11). The most general result in that direction is due to Berestycki and Lions [4 for $N=1$ and $N \geqslant 3$ and Berestycki, Gallouet and Kavian 3 ] for $N=2$.

The assumptions of 3 , 4] when $N \geqslant 2$ are:

(g0) $g$ is continuous and odd,

(g1) if $N \geqslant 3,-\infty<\liminf _{s \rightarrow 0} \frac{g(s)}{s} \leqslant \limsup _{s \rightarrow 0} \frac{g(s)}{s}<0$,

if $N=2,-\infty<\lim _{s \rightarrow 0} \frac{g(s)}{s}:=-\rho<0$,

(g2) if $N \geqslant 3, \lim _{s \rightarrow+\infty} \frac{g(s)}{s^{\frac{N+2}{N-2}}}=0$,

if $N=2, \forall \alpha>0 \exists C_{\alpha}>0$ such that $|g(s)| \leqslant C_{\alpha} e^{\alpha s^{2}} \forall s>0$,

(g3) there exists $\xi_{0}>0$ such that $G\left(\xi_{0}\right)>0$.

It is known that the assumptions (g0)-(g3) are almost optimal to insure the existence of a solution of (11) (see [4, Section 2.2]). In [3, 4] it is proved that for $N \geqslant 2$ and under (g0)-(g3) there exists a positive radial least energy solution $\varphi$ of (1) when the infimum in (4) is taken over the solutions belonging to $H^{1}\left(\mathbb{R}^{N}, \mathbb{R}\right)$. Moreover it is easily deduced from the proofs in [3, 4, that this $\varphi$ is still a least energy solution of (11) when the infimum is, as in (4), taken over the set of all complex-valued solutions. See 10 for a proof of this statement along with a description of the ground states as being of the form $U=e^{i \theta} \tilde{U}$, where $\theta \in \mathbb{R}$ and $\tilde{U}$ is a real positive ground state solution of (1).

In dimension $N=1$, the assumptions in [4 are:

(h0) $g$ is locally Lipschitz continuous and $g(0)=0$,

(h1) there exists $\eta_{0}>0$ such that

$$
G(s)<0 \text { for all } s \in\left(0, \eta_{0}\right), G\left(\eta_{0}\right)=0, g\left(\eta_{0}\right)>0,
$$

and it is proved in 4 that under (h0) the condition (h1) is necessary and sufficient to guarantee the existence of a unique (up to translation) real positive solution of (11). Here also, it can be shown (see [10]) that the least energy levels coincide for complex and real-valued solutions of (11).

Since the pioneering works [2, 8], it is known that the stability/instability of the standing waves is closely linked to additional variational characterizations that the associated ground states enjoy. Recently, in [13] for $N \geqslant 2$ and in [14] for $N=1$, Jeanjean and Tanaka showed that, under the conditions (g0)-(g3) for $N \geqslant 2$ and basically (h0)-(h1) for $N=1$, the functional $S$ admits a mountain-pass geometry. Precisely they show that setting

$$
\Gamma:=\left\{\gamma \in \mathcal{C}\left([0,1], H^{1}\left(\mathbb{R}^{N}\right)\right), \gamma(0)=0, S(\gamma(1))<0\right\},
$$


one has $\Gamma \neq \emptyset$ and

$$
c:=\inf _{\gamma \in \Gamma} \max _{t \in[0,1]} S(\gamma(t))>0 .
$$

Furthermore, they proved that

$$
c=m,
$$

namely that the mountain-pass value gives the least energy level. In fact, the results of [13, 14] are proved within the space $H^{1}\left(\mathbb{R}^{N}, \mathbb{R}\right)$ but it is straightforward to show, see Lemma 16, that this equality also holds in $H^{1}\left(\mathbb{R}^{N}\right)$.

In this paper, we will show, by studying two specific problems, how the ideas and methods developed in [13, 14, can be implemented in the context of instability by blowup for nonlinear Klein-Gordon equations.

First, working with a nonlinearity of power type $\left(f(s)=|s|^{p-1} s\right)$ we find a set of constraints on which the ground states are minimizers of $S$. In particular, this gives an alternative, much simpler proof of results in [17, 22, 23] concerning the derivation of an additional variational characterization of the ground states. Precisely, we prove

Theorem 1. Let $\alpha, \beta \in \mathbb{R}$ be such that

$$
\left\{\begin{array}{l}
\beta<0, \quad \alpha(p-1)-2 \beta \geqslant 0 \text { and } 2 \alpha-\beta(N-2)>0 \\
\text { or } \beta \geqslant 0, \quad \alpha(p-1)-2 \beta \geqslant 0 \text { and } 2 \alpha-\beta N>0 .
\end{array}\right.
$$

Let $\omega \in(-1,1)$ and $\varphi_{\omega} \in H^{1}\left(\mathbb{R}^{N}\right)$ be a ground state solution of

$$
-\Delta \varphi_{\omega}+\left(1-\omega^{2}\right) \varphi_{\omega}-\left|\varphi_{\omega}\right|^{p-1} \varphi_{\omega}=0
$$

where $1<p<2^{\star}-1\left(2^{\star}=+\infty\right.$ if $N=1$ or $2,2^{\star}=\frac{2 N}{N-2}$ if $\left.N \geqslant 3\right)$. Then

$$
S\left(\varphi_{\omega}\right)=\min \left\{S(v) \mid v \in H^{1}\left(\mathbb{R}^{N}\right) \backslash\{0\}, K_{\alpha, \beta}(v)=0\right\},
$$

where

$$
\begin{aligned}
S(v) & :=\frac{1}{2}\|\nabla v\|_{2}^{2}+\frac{1-\omega^{2}}{2}\|v\|_{2}^{2}-\frac{1}{p+1}\|v\|_{p+1}^{p+1}, \\
K_{\alpha, \beta}(v) & :=\frac{2 \alpha-\beta(N-2)}{2}\|\nabla v\|_{2}^{2}+\frac{(2 \alpha-\beta N)\left(1-\omega^{2}\right)}{2}\|v\|_{2}^{2}-\frac{\alpha(p+1)-\beta N}{p+1}\|v\|_{p+1}^{p+1} .
\end{aligned}
$$

The functional $K_{\alpha, \beta}$ is based on the scaling $v_{\lambda}(\cdot):=\lambda^{\alpha} v\left(\lambda^{\beta} \cdot\right)$ for $v \in H^{1}\left(\mathbb{R}^{N}\right)$, precisely, $K_{\alpha, \beta}(v)=\frac{\partial}{\partial \lambda} S\left(v_{\lambda}\right)_{\mid \lambda=1}$. The main idea of the proof of Theorem 1 is to use scaled functions to construct for any $v \in H^{1}\left(\mathbb{R}^{N}\right)$ such that $K_{\alpha, \beta}(v)=0$ a path in $\Gamma$ attaining its maximum at $v$.

It is also of interest to consider a limit case of Theorem 1,

Theorem 2. Let $\alpha, \beta \in \mathbb{R}$ be such that

$$
\left\{\begin{aligned}
\beta<0, & \alpha(p-1)-2 \beta \geqslant 0 \text { and } 2 \alpha-\beta(N-2)=0 \\
\text { or } \quad \beta>0, \quad \alpha(p-1)-2 \beta \geqslant 0 & \text { and } 2 \alpha-\beta N=0 .
\end{aligned}\right.
$$

Let $\omega \in(-1,1)$ and $\varphi_{\omega}$ be a ground state solution of

$$
-\Delta \varphi_{\omega}+\left(1-\omega^{2}\right) \varphi_{\omega}-\left|\varphi_{\omega}\right|^{p-1} \varphi_{\omega}=0 .
$$

Then

$$
S\left(\varphi_{\omega}\right)=\min \left\{S(v) \mid v \in H^{1}\left(\mathbb{R}^{N}\right) \backslash\{0\}, K_{\alpha, \beta}(v)=0\right\} .
$$

Remark 1. Looking to the proofs of Theorems 1 1 and 2, one sees that our theorems remain unchanged when $\left(1-\omega^{2}\right)$ is replaced by any $m>0$. We choose however to present our results in the setting of [17, 22, 23]. 
For $(\alpha, \beta)=\left(\frac{N}{2}, 1\right)$, Theorem 2 gives a simpler proof of a variational characterization of the ground state proved by Berestycki and Cazenave [2] for $1+\frac{4}{N}<p<$ $1+\frac{4}{N-2}$ and by Nawa [19, Proposition 2.5] for $p=1+\frac{4}{N}$. This characterization is at the heart of the classical result of Berestycki and Cazenave [2] dealing with the instability of the ground states of nonlinear Schrödinger equations.

For our second direction of application we consider the instability of the stationary solutions of

$$
u_{t t}-\Delta u=g(u) .
$$

In 1985, Shatah established in 24 that under the conditions (g0)-(g3) the radial ground state solutions associated with the standing waves corresponding to $\omega=0$ are unstable when $N \geqslant 3$. Under stronger hypotheses, but in any dimension and for not necessarily radial solutions, Berestycki and Cazenave [2] had previously proved that these ground states are unstable by blowup in finite time. In 24, instability may occur by blowup in infinite time, in the sense that the $H^{1}\left(\mathbb{R}^{N}\right)$-norm of a solution starting close to a ground state goes to infinity when $t \rightarrow+\infty$. Here, we show that the same result holds when $N=2$.

To ensure that the Cauchy problem is locally well posed for (9) in dimension $N=2$, we require a hypothesis slightly stronger than (g2):

$$
\left(\mathrm{g} 2^{\prime}\right) \forall \alpha>0 \exists C_{\alpha}>0 \text { such that }|g(s)-g(t)| \leqslant C_{\alpha}\left(e^{\alpha s^{2}}+e^{\alpha t^{2}}\right)|s-t| \forall s, t>0 \text {. }
$$

With this hypothesis our nonlinearity is subcritical for the Moser-Trudinger inequality. For the study of the Cauchy problem in the critical case, we refer to the papers [12, 21. Under our assumptions, it is not hard to see that the proofs of 7 , Theorem 6.2.2 and Proposition 6.2.3] can be adapted to give

Proposition 2. Let $N=2$ and assume $(g 0),(g 1)$ and $\left(g 2^{\prime}\right)$. Then for all $\left(u_{0}, v_{0}\right) \in$ $H^{1}\left(\mathbb{R}^{2}\right) \times L^{2}\left(\mathbb{R}^{2}\right)$ there exist $0<T \leqslant+\infty$ and a unique function $u:[0, T) \times \mathbb{R}^{2} \rightarrow \mathbb{C}$ that is a solution of (9) with initial data $\left(u_{0}, v_{0}\right)$ such that

$$
u \in \mathcal{C}\left([0, T), H^{1}\left(\mathbb{R}^{2}\right)\right) \cap \mathcal{C}^{1}\left([0, T), L^{2}\left(\mathbb{R}^{2}\right)\right) \cap \mathcal{C}^{2}\left([0, T), H^{-1}\left(\mathbb{R}^{2}\right)\right),
$$

$E\left(u(t), u_{t}(t)\right)=E\left(u_{0}, v_{0}\right)$ for all $t \in[0, T)$ (energy equality),

if $T<+\infty$, then $\lim _{t \rightarrow T}\left(\|u(t)\|_{H^{1}\left(\mathbb{R}^{2}\right)}+\left\|u_{t}(t)\right\|_{2}\right)=+\infty$ (blow-up alternative).

Here, the energy functional $E$ is defined for $u \in H^{1}\left(\mathbb{R}^{2}\right)$ and $v \in L^{2}\left(\mathbb{R}^{2}\right)$ by

$$
E(u, v):=\frac{1}{2}\|v\|_{2}^{2}+\frac{1}{2}\|\nabla u\|_{2}^{2}-\int_{\mathbb{R}^{2}} G(u) d x .
$$

Remark 3. From the uniqueness result of Proposition 2 it follows that if an initial data $\left(u_{0}, v_{0}\right)$ belongs to $H_{\text {rad }}^{1}\left(\mathbb{R}^{2}\right) \times L_{\text {rad }}^{2}\left(\mathbb{R}^{2}\right)$, then $\left(u(t), u_{t}(t)\right)$ also belongs to $H_{\text {rad }}^{1}\left(\mathbb{R}^{2}\right) \times L_{\text {rad }}^{2}\left(\mathbb{R}^{2}\right)$ for all $t \in[0, T)$.

In what follows, as above, we write $H_{\text {rad }}^{1}\left(\mathbb{R}^{N}\right)\left(\right.$ resp. $\left.L_{\text {rad }}^{2}\left(\mathbb{R}^{N}\right)\right)$ for the space of radial functions of $H^{1}\left(\mathbb{R}^{N}\right)$ (resp. $L^{2}\left(\mathbb{R}^{N}\right)$ ).

Our third main result is the following.

Theorem 3. Assume $N=2,(g 0),(g 1),\left(g 2^{\prime}\right)$ and $(g 3)$. Let $\varphi$ be a radial ground state of (11). Then $\varphi$ viewed as a stationary solution of (9) is strongly unstable. Namely for all $\varepsilon>0$ there exist $u_{\varepsilon} \in H^{1}\left(\mathbb{R}^{2}\right), T_{\varepsilon} \in(0,+\infty]$ and $\left(t_{n}\right) \subset\left(0, T_{\varepsilon}\right)$ such that $\left\|\varphi-u_{\varepsilon}\right\|_{H^{1}\left(\mathbb{R}^{2}\right)}<\varepsilon$ and $\lim _{t_{n} \rightarrow T_{\varepsilon}}\left\|u\left(t_{n}\right)\right\|_{H^{1}\left(\mathbb{R}^{2}\right)}=+\infty$, where $u(t)$ is a solution of (9) with initial data $\left(u_{\varepsilon}, 0\right)$. 
It is still an open question to describe what happens in dimension $N=1$. Indeed, the use of the radial compactness lemma of Strauss (see Lemma 66) restricts our proof to dimensions $N \geqslant 2$. A partial answer is given by the work of Berestycki and Cazenave: for nonlinearities satisfying some additional assumptions (see [2, (H.3)]), the stationary solutions are unstable.

We do hope that the methods developed in this paper will find other areas of applications. In that direction, we mention the work [15] in which the variational characterization $c=m$ derived from [13, 14 is essential to get an alternative, more general proof of the classical result of Berestycki and Cazenave 2] on the instability by blowup for nonlinear Schrödinger equations.

This paper is organized as follows. In Section 2 we prove Theorem 1 and Theorem 2. In Section 3 we prove Theorem 3. The proof that the results of [13, 14] extend to the complex case is given in the Appendix.

\section{VARIATIONAL CHARACTERIZATIONS OF THE GROUND STATES}

In this section, we consider (3) with a power type nonlinearity:

$$
-\Delta \varphi_{\omega}+\left(1-\omega^{2}\right) \varphi_{\omega}-\left|\varphi_{\omega}\right|^{p-1} \varphi_{\omega}=0
$$

where $1<p<1+4 /(N-2)$ and $|\omega|<1$. For this nonlinearity it is known (see 6, Section 8.1] and the references therein) that there exists a unique positive radial ground state $\varphi_{\omega} \in H^{1}\left(\mathbb{R}^{N}, \mathbb{R}\right)$ of $(10)$ and that all ground states are of the form $e^{i \theta} \varphi_{\omega}(\cdot-y)$ for some fixed $\theta \in \mathbb{R}$ and $y \in \mathbb{R}^{N}$. The standing waves $e^{i \omega t} \varphi_{\omega}$ are solutions of the nonlinear Klein-Gordon equation

$$
u_{t t}-\Delta u+u=|u|^{p-1} u
$$

and the natural functional associated with (10) becomes

$$
S(v):=\frac{1}{2}\|\nabla v\|_{2}^{2}+\frac{1-\omega^{2}}{2}\|v\|_{2}^{2}-\frac{1}{p+1}\|v\|_{p+1}^{p+1} .
$$

Various results of instability for the standing waves of (11) were recently proved in [17, 22, 23]. For instance, it was proved in [22] that for any $1<p<1+4 /(N-2)$ the standing wave associated with a ground state of (10) is strongly unstable by blowup if $\omega^{2} \leqslant(p-1) /(p-3)$ and $N \geqslant 3$. In [23], a result of strong instability was showed for the optimal range of parameter $\omega$ in dimension $N \geqslant 2$ (namely $|\omega|<\omega_{c}$, where $\omega_{c}$ was determined in [25]). In both cases, it is central in the proofs that the ground states can be characterized as minimizers on constraints all having the form

$$
\mathscr{K}_{\alpha, \beta}:=\left\{v \in H^{1}\left(\mathbb{R}^{N}\right) \backslash\{0\} \mid K_{\alpha, \beta}(v)=0\right\}
$$

for some $\alpha, \beta \in \mathbb{R}$. Recall that the functional $K_{\alpha, \beta}$ is defined for $v \in H^{1}\left(\mathbb{R}^{N}\right)$ by

$$
\begin{aligned}
K_{\alpha, \beta}(v) & :=\frac{\partial}{\partial \lambda} S\left(\lambda^{\alpha} v\left(\lambda^{\beta} \cdot\right)\right)_{\mid \lambda=1} \\
& =\frac{2 \alpha-\beta(N-2)}{2}\|\nabla v\|_{2}^{2}+\frac{(2 \alpha-\beta N)\left(1-\omega^{2}\right)}{2}\|v\|_{2}^{2}-\frac{\alpha(p+1)-\beta N}{p+1}\|v\|_{p+1}^{p+1} .
\end{aligned}
$$

For example, it is proved in [22] that the ground states are minimizers of $S$ on $\mathscr{K}_{\alpha, \beta}$ for $(\alpha, \beta)=(1,0)$ and $(\alpha, \beta)=(0,-1 / N)$ (see [22, (2.1)]) whereas in [23], the values of $(\alpha, \beta)$ considered are $(\alpha, \beta)=(N / 2,1)$ if $p \geqslant 1+4 / N$ (see [23, (2.11)]) and $(\alpha, \beta)=(2 /(p-1), 1)$ if $p<1+4 / N$ (see [23, (2.18)]). Recently, Liu, Ohta 
and Todorova [17] extended the approach of 22 to the dimensions $N=1,2$. Once more, a main feature of their proof is to minimize $S$ on $\mathscr{K}_{\alpha, \beta}$, but this time with

$$
\alpha=\frac{(p-1)-(p+3) \omega^{2}}{2(p-1) \omega^{2}}, \beta=-1 .
$$

In [17, 22, 23, the proofs that the ground states are minimizers of $S$ on $\mathscr{K}_{\alpha, \beta}$ follow similar schemes. First, one has to show the convergence of a minimizing sequence to some function solving a Lagrange equation. After that, the difficulty is to get rid of the Lagrange multiplier. For each choice of $(\alpha, \beta)$, long computations are involved to prove that the Lagrange multiplier is 0 and to conclude that the obtained function is in fact a solution of (10).

Our proof of Theorem 1 relies on the following lemma. We recall that $\Gamma$ is defined in (5).

Lemma 4. Let $\alpha, \beta \in \mathbb{R}$ satisfy (7). Then for all $v \in \mathscr{K}_{\alpha, \beta}$ we can construct a path $\gamma$ in $\Gamma$ such that

$$
\max _{t \in[0,1]} S(\gamma(t))=S(v) .
$$

Proof. Let $v \in \mathscr{K}_{\alpha, \beta}$. For all $\lambda \in(0,+\infty)$ we define $v_{\lambda} \in H^{1}\left(\mathbb{R}^{N}\right)$ by $v_{\lambda}(\cdot):=$ $\lambda^{\alpha} v\left(\lambda^{\beta} \cdot\right)$. The idea is to construct the path such that $\gamma(t)=v_{C t}$ for some $C>0$.

The first thing to check is that we can extend $\gamma$ at 0 by continuity. Namely, we must show that under (7) we have $\lim _{\lambda \rightarrow 0}\left\|v_{\lambda}\right\|_{H^{1}\left(\mathbb{R}^{N}\right)}=0$. This is immediate if we remark that

$$
\left\|v_{\lambda}\right\|_{H^{1}\left(\mathbb{R}^{N}\right)}^{2}=\lambda^{2 \alpha-\beta(N-2)}\|\nabla v\|_{2}^{2}+\lambda^{2 \alpha-\beta N}\|v\|_{2}^{2},
$$

and that (7) implies

$$
2 \alpha-\beta(N-2)>0 \text { and } 2 \alpha-\beta N>0 .
$$

The next step is to prove that $\lambda \rightarrow S\left(v_{\lambda}\right)$ increases for $\lambda \in(0,1)$, attains its maximum at $\lambda=1$ and decreases toward $-\infty$ on $(1,+\infty)$. We have

$$
S\left(v_{\lambda}\right)=\frac{\lambda^{2 \alpha-\beta(N-2)}}{2}\|\nabla v\|_{2}^{2}+\frac{\left(1-\omega^{2}\right) \lambda^{2 \alpha-\beta N}}{2}\|v\|_{2}^{2}-\frac{\lambda^{(p+1) \alpha-\beta N}}{p+1}\|v\|_{p+1}^{p+1}
$$

and from easy computations it becomes

$$
\begin{aligned}
\lambda^{-(2 \alpha-\beta N-1)} \frac{\partial}{\partial \lambda} S\left(v_{\lambda}\right)= & \lambda^{2 \beta} \frac{2 \alpha-\beta(N-2)}{2}\|\nabla v\|_{2}^{2}+\frac{(2 \alpha-\beta N)\left(1-\omega^{2}\right)}{2}\|v\|_{2}^{2} \\
& -\lambda^{\alpha(p-1)} \frac{\alpha(p+1)-\beta N}{p+1}\|v\|_{p+1}^{p+1} .
\end{aligned}
$$

Therefore, if $\alpha$ and $\beta$ satisfy

$$
\left\{\begin{array}{l}
\quad \beta \neq 0 \text { and } \quad \alpha(p-1) \geqslant 2 \beta \\
\text { or } \quad \beta=0 \text { and } \quad \alpha(p-1)>0,
\end{array}\right.
$$

then

$$
\left\{\begin{array}{l}
\frac{\partial}{\partial \lambda} S\left(v_{\lambda}\right)>0 \text { for } \lambda \in(0,1), \\
\frac{\partial}{\partial \lambda} S\left(v_{\lambda}\right)<0 \text { for } \lambda \in(1,+\infty), \\
\lim _{\lambda \rightarrow+\infty} S\left(v_{\lambda}\right)=-\infty .
\end{array}\right.
$$

Since $\alpha>0$ when $\beta=0$ in (7) it is clear that (12) holds under (7). 
Finally, choosing $C$ large enough to have $S\left(v_{C}\right)<0$ and defining $\gamma:[0,1] \mapsto$ $H^{1}\left(\mathbb{R}^{N}\right)$ by

$$
\gamma(0):=0 \text { and } \gamma(t):=v_{t C}
$$

we have a path satisfying the conclusion of the lemma.

Proof of Theorem 1. Let $\varphi_{\omega}$ be a least energy solution of (10) for $|\omega|<1$. From Lemma 16 we know that

$$
c=m,
$$

where $m$ is the least energy level and $c$ the mountain-pass value (see (4) and (6) for the definitions of $m$ and $c$ ). Since $\varphi_{\omega}$ is a solution of (10), $\varphi_{\omega} \in \mathcal{C}^{1}$ and $\varphi_{\omega}$, $\nabla \varphi_{\omega}$ are exponentially decaying at infinity (see, for example, [6, Theorem 8.1.1]); in particular, $x . \nabla \varphi_{\omega} \in H^{1}\left(\mathbb{R}^{N}\right)$, and

$$
K_{\alpha, \beta}\left(\varphi_{\omega}\right)=\left.\frac{\partial}{\partial \lambda} S\left(\lambda^{\alpha} \varphi_{\omega}\left(\lambda^{\beta} \cdot\right)\right)\right|_{\lambda=1}=\left\langle S^{\prime}\left(\varphi_{\omega}\right), \alpha \varphi_{\omega}+\beta x . \nabla \varphi_{\omega}\right\rangle=0 .
$$

Thus $\varphi_{\omega} \in \mathscr{K}_{\alpha, \beta}$ and

$$
\min \left\{S(v) \mid v \in \mathscr{K}_{\alpha, \beta}\right\} \leqslant S\left(\varphi_{\omega}\right)=c .
$$

Conversely, it follows from Lemma 4 that

$$
c \leqslant \min \left\{S(v) \mid v \in \mathscr{K}_{\alpha, \beta}\right\} .
$$

Combining (13) and (14) finishes the proof.

We now turn to the proof of Theorem 2. It follows the same lines as for Theorem 1. find a path reaching its maximum on the constraint $\mathscr{K}_{\alpha, \beta}$ and use the equality $c=m$. The main difference is in the way we construct the path: we still want to use the scaled functions $v_{\lambda}$, but their $H^{1}\left(\mathbb{R}^{N}\right)$-norm no longer converges to 0 as $\lambda \rightarrow 0$. This difficulty is overcome by gluing to $\left\{v_{\lambda}\right\}_{\lambda>\lambda_{0}}$ a path linking 0 to $v_{\lambda_{0}}$ for $\lambda_{0}$ suitably chosen. The lemma is

Lemma 5. Let $\alpha, \beta \in \mathbb{R}$ satisfy (8). Then for all $v \in \mathscr{K}_{\alpha, \beta}$ we can construct a path $\gamma$ in $\Gamma$ such that

$$
\max _{t \in[0,1]} S(\gamma(t))=S(v)
$$

Proof. Let $v \in \mathscr{K}_{\alpha, \beta}$ and $v_{\lambda_{0}}(\cdot):=\lambda_{0}^{\alpha} v\left(\lambda_{0}^{\beta} \cdot\right)$ for some $\lambda_{0} \in(0,1)$ whose value will be fixed later. Let $C>0$ be such that $S\left(v_{C}\right)<0$ and consider the curves

$$
\begin{aligned}
& \Lambda_{1}:=\left\{v_{\lambda} \mid \lambda \in\left[\lambda_{0}, C\right]\right\}, \\
& \Lambda_{2}:=\left\{t v_{\lambda_{0}} \mid t \in[0,1]\right\} .
\end{aligned}
$$

To get a path as desired, we will glue the two curves $\Lambda_{1}$ and $\Lambda_{2}$. It is clear that as in the proof of Lemma 4, $S$ attains its maximum on $\Lambda_{1}$ at $v$. Thus the only thing we have to check is that $t \mapsto S\left(t v_{\lambda_{0}}\right)$ is increasing on $[0,1]$.

We have

$$
\frac{\partial}{\partial t} S\left(t v_{\lambda_{0}}\right)=t\left(\left\|\nabla v_{\lambda_{0}}\right\|_{2}^{2}+\left(1-\omega^{2}\right)\left\|v_{\lambda_{0}}\right\|_{2}^{2}-t^{p-1}\left\|v_{\lambda_{0}}\right\|_{p+1}^{p+1}\right) .
$$

If $\beta>0$ and $\alpha=\beta N / 2$ (see (8) ), then $\lambda_{0} \rightarrow\left\|v_{\lambda_{0}}\right\|_{2}$ is constant. If $\beta<0$ and $\alpha=\beta(N-2) / 2$, then $\lambda_{0} \rightarrow\left\|\nabla v_{\lambda_{0}}\right\|_{2}$ is constant. Moreover, we have in any case

$$
\lim _{\lambda_{0} \rightarrow 0}\left\|v_{\lambda_{0}}\right\|_{p+1}^{p+1}=0
$$


Therefore, if $\lambda_{0} \in(0,1)$ is small enough we have

$$
\frac{\partial}{\partial t} S\left(t v_{\lambda_{0}}\right)>0 \text { for } t \in(0,1) .
$$

Defining $\gamma:[0,1] \mapsto H^{1}\left(\mathbb{R}^{N}\right)$ by

$$
\left\{\begin{array}{l}
\gamma(t)=\frac{C t}{\lambda_{0}} v_{\lambda_{0}} \text { for } t \in\left[0, \frac{\lambda_{0}}{C}\right) \\
\gamma(t)=v_{C t} \text { for } t \in\left[\frac{\lambda_{0}}{C}, 1\right]
\end{array}\right.
$$

gives us the desired path.

Proof of Theorem 2. The proof is identical to the proof of Theorem 1 with Lemma 4 replaced by Lemma 5 .

\section{Orbital instability FOR A GENERALIZED NONLINEAR KLEIN-GORDON EQUATION}

In this section, we consider the nonlinear Klein-Gordon equation with a general nonlinearity

$$
u_{t t}-\Delta u=g(u) .
$$

We assume throughout the section that (g0), (g1), (g2') and (g3) hold. Observe nevertheless that Lemmas 7 and 10 hold true just under (g0)-(g3).

In [24], Shatah proved that for $N \geqslant 3$, under (g0)-(g3), the radial ground state solutions of

$$
-\Delta \varphi=g(\varphi), \quad u \in H^{1}\left(\mathbb{R}^{N}\right)
$$

viewed as stationary solutions of (15) are unstable in the sense of Theorem 3 .

The restriction to $N \geqslant 3$ has its origin in, at least, two reasons.

First, one needs to control the decay in $|x|$ of $u(t, x)$ uniformly in $t$. This appears in the proof of Lemma 14. For this control, the following compactness lemma due to Strauss [26] is used.

Lemma 6. Let $N \geqslant 2$ and $v \in H_{\mathrm{rad}}^{1}\left(\mathbb{R}^{N}\right)$. Then

$$
|v(x)| \leqslant C|x|^{\frac{1-N}{2}}\|u\|_{H^{1}\left(\mathbb{R}^{N}\right)} \text { a.e. }
$$

with $C$ independent of $x$ and $u$. In particular, the following injection is compact:

$$
H_{\text {rad }}^{1}\left(\mathbb{R}^{N}\right) \hookrightarrow L^{q}\left(\mathbb{R}^{N}\right) \text { for } 2<q<2^{\star},
$$

where $2^{\star}=\frac{2 N}{N-2}$ if $N \geqslant 3$ and $2^{\star}=+\infty$ if $N=2$.

Actually, to use this lemma only $N \geqslant 2$ is necessary.

A second reason for the restriction $N \geqslant 3$ in 24 is found in the use of a constraint based on the Pohozaev identity to derive a variational characterization of the ground states, to define an invariant set, and, most important, to choose suitable initial data close to the ground states. Thanks to our approach, we arrive at this second point only by requiring $N \geqslant 2$.

Our proof will make use of the following variational characterization of the ground states. 
Lemma 7. Let $\varphi \in H^{1}\left(\mathbb{R}^{2}\right)$ be a ground state of (16). Then

$$
S(\varphi)=m=\min _{v \in \mathscr{P}} S(v)>0
$$

where

$$
\mathscr{P}:=\left\{v \in H^{1}\left(\mathbb{R}^{2}\right) \backslash\{0\} \mid P(v)=0\right\}
$$

with $P(v):=\int_{\mathbb{R}^{2}} G(v) d x$ for $v \in H^{1}\left(\mathbb{R}^{2}\right)$.

This lemma was proved in $\left[3\right.$ when $v \in H^{1}\left(\mathbb{R}^{2}, \mathbb{R}\right)$. It can trivially be extended to $v \in H^{1}\left(\mathbb{R}^{2}, \mathbb{C}\right)$; see [10].

Remark 8. The functional $P$ is related to the so-called Pohozaev identity (see 4, Proposition 1], [26]): for $N \geqslant 1$, any solution $v \in H^{1}\left(\mathbb{R}^{N}\right)$ of (16) satisfies

$$
\frac{N-2}{2}\|\nabla v\|_{2}^{2}-N \int_{\mathbb{R}^{N}} G(v) d x=0 .
$$

A main feature of the dimension $N=2$ is that we lose the control on the $L^{2}\left(\mathbb{R}^{N}\right)$ norm of $\nabla v$.

Remark 9. For $N \geqslant 3$, Shatah also showed that the radial ground states are minimizers of $S$ among all nontrivial functions satisfying the Pohozaev identity (see [24, Proposition 1.5]). His method consists in proving that the minimization problem has a solution and then in eliminating the Lagrange multiplier. In fact, as is done in [13, Lemma 3.1], a shorter proof can be performed by simply establishing a correspondence with a minimization problem already solved in [4.

The scheme of the proof is the following: first, define a set $\mathcal{I} \subset H_{\text {rad }}^{1}\left(\mathbb{R}^{2}\right) \times$ $L_{\text {rad }}^{2}\left(\mathbb{R}^{2}\right)$ such that any solution of (15) with initial data in $\mathcal{I}$ stays in $\mathcal{I}$ for all time and blows up; then prove that the ground states can be approximated by functions in $\mathcal{I}$.

Let $\mathcal{I}$ be defined by

$$
\mathcal{I}:=\left\{u \in H_{\mathrm{rad}}^{1}\left(\mathbb{R}^{2}\right) \backslash\{0\}, v \in L_{\mathrm{rad}}^{2}\left(\mathbb{R}^{2}\right) \mid E(u, v)<m, P(u)>0\right\} .
$$

We begin by proving an equivalence between two variational problems.

Lemma 10. We have

$$
0<m=\min _{v \in \mathscr{P}} S(v)=\min \left\{T(v) \mid v \in H^{1}\left(\mathbb{R}^{2}\right) \backslash\{0\}, P(v) \geqslant 0\right\},
$$

where $T(v):=\frac{1}{2}\|\nabla v\|_{2}^{2}$.

Proof. Let $v \in H^{1}\left(\mathbb{R}^{2}\right)$. If $v \in \mathscr{P}$, then $v$ satisfies $T(v)=S(v)$ and thanks to Lemma 7. $T(v) \geqslant m$. Suppose that $P(v)>0$. For $\lambda>0$, define $v_{\lambda}(\cdot):=\lambda v(\lambda \cdot)$. We claim that there exists $\lambda_{0}<1$ such that $P\left(v_{\lambda_{0}}\right)=0$. Indeed, by (g1)-(g2), for all $\alpha>0$ there exists $C_{\alpha}>0$ such that for $s>0$,

$$
g(s) \leqslant \frac{-\rho s}{2}+2 s \alpha C_{\alpha} e^{\alpha s^{2}} .
$$

We recall that $\rho>0$ is given in $(\mathrm{g} 1)$ by $\lim _{s \rightarrow 0} g(s) s^{-1}=-\rho$. Therefore, for $s>0$ we have

$$
G(s) \leqslant \frac{-\rho s^{2}}{4}+C_{\alpha}\left(e^{\alpha s^{2}}-1\right)
$$


and

$$
\int_{\mathbb{R}^{2}} G\left(v_{\lambda}\right) \leqslant \frac{-\rho\left\|v_{\lambda}\right\|_{2}^{2}}{4}+C_{\alpha} \int_{\mathbb{R}^{2}}\left(e^{\alpha v_{\lambda}^{2}}-1\right) d x .
$$

We remark that $\left\|v_{\lambda}\right\|_{2}^{2}=\|v\|_{2}^{2}$ and

$$
\int_{\mathbb{R}^{2}}\left(e^{\alpha v_{\lambda}^{2}}-1\right) d x=\lambda^{-2} \int_{\mathbb{R}^{2}}\left(e^{\alpha \lambda^{2} v^{2}}-1\right) d x
$$

For $\lambda<1$ we have

$$
\lambda^{-2}\left(e^{\alpha \lambda^{2} v^{2}(x)}-1\right)<e^{\alpha v^{2}(x)}-1 \text { for all } x \in \mathbb{R}^{2},
$$

and by the Moser-Trudinger inequality (see [1, Theorem 8.25]) there exists $\alpha>0$ such that $\left(e^{\alpha v^{2}}-1\right) \in L^{1}\left(\mathbb{R}^{2}\right)$. Hence, Lebesgue's Theorem gives

$$
\int_{\mathbb{R}^{2}}\left(e^{\alpha v_{\lambda}^{2}}-1\right) d x \rightarrow 0 \text { when } \lambda \rightarrow 0 .
$$

Coming back to (18) this means that

$$
\int_{\mathbb{R}^{2}} G\left(v_{\lambda}\right)<0 \text { for } \lambda>0 \text { small enough, }
$$

and by continuity of $P$ this proves the claim.

Now, we have

$$
\inf _{u \in \mathscr{P}} S(u) \leqslant S\left(v_{\lambda_{0}}\right)=T\left(v_{\lambda_{0}}\right)=\lambda_{0}^{2} T(v)<T(v)
$$

and the lemma is proved.

Next we prove that the set $\mathcal{I}$ is invariant under the flow of (15).

Lemma 11. Let $\left(u_{0}, v_{0}\right) \in \mathcal{I}, 0<T \leqslant+\infty$ and let $u(t)$ be a solution of (15) on $[0, T)$ with initial data $\left(u_{0}, v_{0}\right)$. Then $\left(u(t), u_{t}(t)\right) \in \mathcal{I}$ for all $t \in[0, T)$.

Proof. Assume by contradiction that there exists $t_{1} \in(0, T)$ such that $P\left(u\left(t_{1}\right)\right) \leqslant 0$. By continuity there exists $t_{0} \in\left(0, t_{1}\right]$ such that $P\left(u\left(t_{0}\right)\right)=0$. Since, by Lemma 7 . $T(v) \geqslant m$ on the set $\left\{v \in H^{1}\left(\mathbb{R}^{2}\right) \backslash\{0\}, P(v)>0\right\}$ it follows by continuity that $T\left(u\left(t_{0}\right)\right) \geqslant m>0$ and thus $u\left(t_{0}\right) \neq 0$. Namely $u\left(t_{0}\right) \in \mathscr{P}$. Now, recall that

$$
S\left(u\left(t_{0}\right)\right) \leqslant E\left(u\left(t_{0}\right), u_{t}\left(t_{0}\right)\right) .
$$

By the energy equality, this implies that

$$
S\left(u\left(t_{0}\right)\right)<m,
$$

which is a contradiction with Lemma 7

The following lemma is a key step in the proof.

Lemma 12. Let $\left(u_{0}, v_{0}\right) \in \mathcal{I}$ and let $u(t)$ be an associated solution of (15) in $[0, T)$. Then there exists $\delta>0$ such that $P(u(t))>\delta$ for all $t \in[0, T)$.

Proof. Indeed, assume by contradiction that there exists a sequence $\left(t_{n}\right)$ such that $P\left(u\left(t_{n}\right)\right) \rightarrow 0$ as $n \rightarrow+\infty$. Then

$$
\begin{aligned}
T\left(u\left(t_{n}\right)\right) & =S\left(u\left(t_{n}\right)\right)+P\left(u\left(t_{n}\right)\right) \\
& \leqslant E\left(u\left(t_{n}\right), u_{t}\left(t_{n}\right)\right)+P\left(u\left(t_{n}\right)\right) .
\end{aligned}
$$

By the energy equality in Proposition 2 this implies

$$
T\left(u\left(t_{n}\right)\right) \leqslant E\left(u_{0}, v_{0}\right)+P\left(u\left(t_{n}\right)\right)
$$


and thus

$$
T\left(u\left(t_{n}\right)\right) \leqslant m+P\left(u\left(t_{n}\right)\right)-\nu
$$

with $\nu:=m-E\left(u_{0}, v_{0}\right)>0$ since $\left(u_{0}, v_{0}\right) \in \mathcal{I}$. For $n$ large enough we have

$$
0 \leqslant P\left(u\left(t_{n}\right)\right)<\nu / 2
$$

and thus (19) gives

$$
T\left(u\left(t_{n}\right)\right)<m-\frac{\nu}{2}
$$

which contradicts the result of Lemma 10.

The proof of Theorem 3 relies on the following proposition.

Proposition 13. Let $\left(u_{0}, v_{0}\right) \in \mathcal{I}$ and let $u(t)$ be an associated solution of (15) on $[0, T)$. Then there exists $\left(t_{n}\right) \subset(0, T)$ such that $\lim _{t_{n} \rightarrow T}\left\|u\left(t_{n}\right)\right\|_{H^{1}\left(\mathbb{R}^{2}\right)}=+\infty$.

Proposition 13 is obtained with the help of the following lemma.

Lemma 14. Let $\left(u_{0}, v_{0}\right) \in \mathcal{I}$ and let $u(t)$ be an associated solution of (15) on $[0,+\infty)$. Suppose in addition that $\|u(t)\|_{H^{1}\left(\mathbb{R}^{N}\right)}<K$ on $[0,+\infty)$ for some constant $K<+\infty$. Then there exist $C, \nu>0$ such that for all $t \in[0,+\infty)$,

$$
\nu t \leqslant C\left(1+\left\|u_{t}\right\|_{2}\|\nabla u\|_{2}\right) .
$$

Proof. The proof relies on the identity

$$
\left\|u_{t}\right\|_{2}^{2}+2 \int_{\mathbb{R}^{2}} G(u) d x=-\operatorname{Re} \frac{\partial}{\partial t} \int_{\mathbb{R}^{2}} u_{t} x \cdot \nabla \bar{u} d x,
$$

which is formally obtained by multiplying (15) by $x \cdot \nabla \bar{u}$. However, it is not clear that the integral in the right member is well-defined; thus we shall use a truncated version of this identity. It is convenient to work in the radial variable (which is possible because the initial data in $\mathcal{I}$ are radial and this implies that $u(t)$ is also radial; see Remark (3). Thus (15) becomes

$$
u_{t t}-\frac{1}{r} u^{\prime}-u^{\prime \prime}=g(u),
$$

where the space variable $x \in \mathbb{R}^{2}$ is replaced by $r \in(0,+\infty)$ and the ' and " stand for the first and second derivatives with respect to $r$. Inspired by the truncation function used in [23], we define for $\varepsilon>0$,

$$
\Phi_{\varepsilon}(r):= \begin{cases}2 & \text { if } 0 \leqslant r \leqslant 1 \\ 2-2 \varepsilon \ln (r) & \text { if } 1<r \leqslant e^{\varepsilon^{-1}} \\ 0 & \text { if } r>e^{\varepsilon^{-1}}\end{cases}
$$

and $\Psi_{\varepsilon}(r):=\frac{1}{r} \int_{0}^{r} s \Phi_{\varepsilon}(s) d s$. We claim that $\Phi_{\varepsilon}$ and $\Psi_{\varepsilon}$ enjoy the following properties:

$$
\begin{aligned}
& \Psi_{\varepsilon}^{\prime}(r)+\frac{1}{r} \Psi_{\varepsilon}(r)=\Phi_{\varepsilon}(r) \text { for all } r>0, \\
& \left\|\Psi_{\varepsilon}^{\prime}-\frac{1}{r} \Psi_{\varepsilon}\right\|_{\infty}<\varepsilon .
\end{aligned}
$$

Indeed, (21) follows from a simple computation:

$$
\Psi_{\varepsilon}^{\prime}+\frac{1}{r} \Psi_{\varepsilon}=-\frac{1}{r^{2}} \int_{0}^{r} s \Phi_{\varepsilon}(s) d s+\frac{r \Phi_{\varepsilon}(r)}{r}+\frac{1}{r^{2}} \int_{0}^{r} s \Phi_{\varepsilon}(s) d s=\Phi_{\varepsilon}(r) .
$$


For (22), we argue as follows. By integration by parts, we have

$$
2 r \Psi_{\varepsilon}(r)=r^{2} \Phi_{\varepsilon}(r)-\int_{0}^{r} s^{2} \Phi_{\varepsilon}^{\prime}(s) d s
$$

By (21) we have

$$
\Psi_{\varepsilon}^{\prime}(r)-\frac{1}{r} \Psi_{\varepsilon}(r)=\Phi_{\varepsilon}(r)-\frac{2}{r} \Psi_{\varepsilon}(r) .
$$

Combining (23) and (24) gives

$$
\Psi_{\varepsilon}^{\prime}(r)-\frac{1}{r} \Psi_{\varepsilon}(r)=\frac{1}{r^{2}} \int_{0}^{r} s^{2} \Phi_{\varepsilon}^{\prime}(s) d s
$$

Now,

$$
\Phi_{\varepsilon}^{\prime}(r):=\left\{\begin{array}{lll}
0 & \text { if } & 0 \leqslant r \leqslant 1 \text { or if } r>e^{\varepsilon^{-1}}, \\
-\frac{2 \varepsilon}{r} & \text { if } & 1<r \leqslant e^{\varepsilon^{-1}} .
\end{array}\right.
$$

Thus if $0<r \leqslant 1$, then $\Psi_{\varepsilon}^{\prime}(r)-\frac{1}{r} \Psi_{\varepsilon}(r)=0$. If $1<r \leqslant e^{\varepsilon^{-1}}$, then

$$
\Psi_{\varepsilon}^{\prime}(r)-\frac{1}{r} \Psi_{\varepsilon}(r)=-\frac{1}{r^{2}} \int_{1}^{r} 2 \varepsilon s d s=-\varepsilon\left(\frac{r^{2}-1}{r^{2}}\right),
$$

which implies $\left|\Psi_{\varepsilon}^{\prime}(r)-\frac{1}{r} \Psi_{\varepsilon}(r)\right| \leqslant \varepsilon$. Finally, if $r>e^{\varepsilon^{-1}}$, then

$$
\Psi_{\varepsilon}^{\prime}(r)-\frac{1}{r} \Psi_{\varepsilon}(r)=-\frac{1}{r^{2}} \int_{1}^{e^{\varepsilon^{-1}}} 2 \varepsilon s d s=-\varepsilon\left(\frac{e^{2 \varepsilon^{-1}}-1}{r^{2}}\right),
$$

which also implies $\left|\Psi_{\varepsilon}^{\prime}(r)-\frac{1}{r} \Psi_{\varepsilon}(r)\right| \leqslant \varepsilon$ and finishes the proof of (22).

We multiply (20) by $\Psi_{\varepsilon}(r) \bar{u}^{\prime} r$ and integrate over $[0,+\infty)$ to get after integration by parts,

$$
\begin{aligned}
\frac{1}{2} \int_{0}^{+\infty}\left|u_{t}\right|^{2} & \Phi_{\varepsilon}(r) r d r+\frac{1}{2} \int_{0}^{+\infty}\left|u^{\prime}\right|^{2}\left(\Psi_{\varepsilon}^{\prime}(r)-\frac{1}{r} \Psi_{\varepsilon}(r)\right) r d r \\
+ & \int_{0}^{+\infty} G(u) \Phi_{\varepsilon}(r) r d r=-\operatorname{Re} \frac{\partial}{\partial t} \int_{0}^{+\infty} u_{t} \Psi_{\varepsilon}(r) \bar{u}^{\prime} r d r .
\end{aligned}
$$

Since $u(t)$ is bounded in $H^{1}\left(\mathbb{R}^{2}\right)$, by (22) we have, for $\varepsilon>0$ small enough,

$$
\left.\left|\frac{1}{2} \int_{0}^{+\infty}\right| u^{\prime}\right|^{2}\left(\Psi_{\varepsilon}^{\prime}(r)-\frac{1}{r} \Psi_{\varepsilon}(r)\right) r d r \mid<\frac{\delta}{2},
$$

where $\delta>0$ is given by Lemma 12. Also, since $\Phi_{\varepsilon} \geqslant 0$, we have

$$
\frac{1}{2} \int_{0}^{+\infty}\left|u_{t}\right|^{2} \Phi_{\varepsilon}(r) r d r \geqslant 0 .
$$

By (g1), there exists $\eta>0$ such that $G(s) \leqslant 0$ for $0 \leqslant s \leqslant \eta$. Thus since $\|u(t)\|_{H^{1}\left(\mathbb{R}^{N}\right)}<C$ on $[0,+\infty)$, using Lemma 6 , there exists $M<\infty$ such that

$$
|u(t, r)| \leqslant \eta \text { for all } r \geqslant M \text { and for all } t \in[0,+\infty) .
$$

This implies that

$$
\int_{M}^{+\infty} G(u) \Phi_{\varepsilon}(r) r d r \geqslant \int_{M}^{+\infty} 2 G(u) r d r .
$$

Clearly also since $\|u(t)\|_{H^{1}\left(\mathbb{R}^{N}\right)}<C$ on $[0,+\infty)$,

$$
\int_{0}^{M} G(u) \Phi_{\varepsilon}(r) r d r \rightarrow \int_{0}^{M} 2 G(u) r d r \text { when } \varepsilon \rightarrow 0 .
$$


Thus for $\varepsilon>0$ small enough, we have

$$
\int_{0}^{+\infty} G(u) \Phi_{\varepsilon}(r) r d r \geqslant \frac{3 \delta}{2},
$$

where $\delta>0$ is still given by Lemma 12, Combining (25)-(28) gives

$$
\delta \leqslant-\operatorname{Re} \frac{\partial}{\partial t} \int_{0}^{+\infty} u_{t} \Psi_{\varepsilon}(r) \bar{u}^{\prime} r d r .
$$

We integrate in time to obtain

$$
\delta t \leqslant-\operatorname{Re} \int_{0}^{+\infty} u_{t} \Psi_{\varepsilon}(r) \bar{u}^{\prime} r d r+\operatorname{Re} \int_{0}^{+\infty} v_{0} \Psi_{\varepsilon}(r) \overline{u_{0}}{ }^{\prime} r d r
$$

and by the Cauchy-Schwarz inequality we get

$$
\delta t \leqslant C\left(1+\left\|u_{t}\right\|_{2}\|\nabla u\|_{2}\right),
$$

which ends the proof of the lemma.

Proof of Proposition 13. First, if $T<+\infty$, the assertion of Proposition 13 is just the blow-up alternative in Proposition 2. Thus we suppose $T=+\infty$ and, by contradiction, that $\left(\|u(t)\|_{H^{1}\left(\mathbb{R}^{N}\right)}\right)$ is bounded. By Lemma 14, there exists $\nu>0$ such that

$$
\nu t \leqslant C\left(1+\left\|u_{t}\right\|_{2}\|\nabla u\|_{2}\right) .
$$

But, from the energy equality, we have

$$
\frac{1}{2}\left\|u_{t}\right\|_{2}^{2}=E\left(u_{0}, v_{0}\right)-\frac{1}{2}\|\nabla u\|_{2}^{2}+\int_{\mathbb{R}^{2}} G(u) d x,
$$

and since $u(t)$ is bounded in $H^{1}\left(\mathbb{R}^{2}\right)$ this implies that $\left\|u_{t}(t)\right\|_{2}$ is bounded. By assumption, $\|\nabla u(t)\|_{2}$ is also bounded; therefore, for $t$ large enough we reach a contradiction in (29).

In dimension $N \geqslant 3$, it is easily seen that for $\lambda<1$ the dilatation of a ground state $\varphi_{\lambda}(\cdot):=\varphi(\dot{\bar{\lambda}})$ gives a sequence of initial data in $\mathcal{I}$ converging to this ground state. This property, combined with the equivalent of Proposition 13, gives immediately the instability of the ground states in [24]. This is not the case any more in dimension $N=2$ where the dilatation $\varphi_{\lambda}(\cdot):=\varphi(\dot{\bar{\lambda}})$ leaves $\mathscr{P}$ and $T$ invariant. To overcome this difficulty, we borrow and adapt an idea of [5, Proposition 2] which consists in using separately (and successively) a dilatation and a scaling to get initial data in $\mathcal{I}$ close to the ground states.

Lemma 15. Let $\varphi \in H^{1}\left(\mathbb{R}^{2}\right)$ be a ground state of (16). For all $\varepsilon>0$ there exists $\varphi_{\varepsilon}$ such that

$$
\left\|\varphi-\varphi_{\varepsilon}\right\|_{H^{1}\left(\mathbb{R}^{2}\right)}<\varepsilon, S\left(\varphi_{\varepsilon}\right)<S(\varphi), P\left(\varphi_{\varepsilon}\right)>0
$$

Proof. For $\lambda, \mu>0$ consider $\varphi_{\lambda, \mu}(\cdot):=\lambda \varphi(\dot{\dot{\mu}})$. Then

$$
\frac{\partial}{\partial \lambda} S\left(\varphi_{\lambda, \mu}\right)=\lambda^{2}\|\nabla \varphi\|_{2}^{2}-\mu^{2} \int_{\mathbb{R}^{2}} g(\lambda \varphi) \bar{\varphi} d x
$$

Multiplying (16) by $\bar{\varphi}$ and integrating gives us

$$
\|\nabla \varphi\|_{2}^{2}=\int_{\mathbb{R}^{2}} g(\varphi) \bar{\varphi} d x
$$


Hence, for $\lambda=1$ we get

$$
\left.\frac{\partial}{\partial \lambda} S\left(\varphi_{\lambda, \mu}\right)\right|_{\lambda=1}=\left(1-\mu^{2}\right)\|\nabla \varphi\|_{2}^{2}
$$

Thus, for all $\mu>1$, there exists $\lambda_{\mu}>0$ such that

$$
\frac{\partial}{\partial \lambda} S\left(\varphi_{\lambda, \mu}\right)<0 \text { for } \lambda \in\left(1-\lambda_{\mu}, 1+\lambda_{\mu}\right)
$$

and therefore

$$
S\left(\varphi_{\lambda, \mu}\right)<S(\varphi) \text { for } \lambda \in\left(1,1+\lambda_{\mu}\right) .
$$

Now,

$$
\left.\frac{\partial}{\partial \lambda} P\left(\varphi_{\lambda, \mu}\right)\right|_{\lambda=1}=\mu^{2} \int_{\mathbb{R}^{2}} g(\varphi) \bar{\varphi} d x=\mu^{2}\|\nabla \varphi\|_{2}^{2}>0 .
$$

Thus, for all $\mu>0$, there exists $\lambda_{\mu}$ such that

$$
\frac{\partial}{\partial \lambda} P\left(\varphi_{\lambda, \mu}\right)>0 \text { for } \lambda \in\left(1-\lambda_{\mu}, 1+\lambda_{\mu}\right)
$$

and therefore

$$
P\left(\varphi_{\lambda, \mu}\right)>0 \text { for } \lambda \in\left(1,1+\lambda_{\mu}\right) .
$$

Finally, from (30)-(31), for $\lambda, \mu>1$ close enough to 1 we get the desired result.

Proof of Theorem 3, Let $\varepsilon>0$ and let $\varphi_{\varepsilon}$ be as given in Lemma 15. Then $\left(\varphi_{\varepsilon}, 0\right)$ satisfies

$$
E\left(\varphi_{\varepsilon}, 0\right)=S\left(\varphi_{\varepsilon}\right)<m \text { and } P\left(\varphi_{\varepsilon}\right)>0,
$$

namely $\left(\varphi_{\varepsilon}, 0\right) \in \mathcal{I}$. Theorem 3 follows now from Proposition 13

\section{Appendix}

Lemma 16. Let $m$ denote the least energy level defined in (4) and $c$ the mountainpass level defined in (6). Then $m=c$.

Proof. In [13, Theorem 0.2] for $N \geqslant 2$ and [14, Theorem 1.2] for $N=1$ it is shown that when the class $\Gamma$ is replaced by

$$
\tilde{\Gamma}:=\left\{\gamma \in \mathcal{C}\left([0,1], H^{1}\left(\mathbb{R}^{N}, \mathbb{R}\right)\right), \gamma(0)=0, S(\gamma(1))<0\right\}
$$

one has

$$
\tilde{c}:=\inf _{\gamma \in \tilde{\Gamma}} \max _{t \in[0,1]} S(\gamma(t))=\tilde{m},
$$

where $\tilde{m}$ is the least energy level among real-valued solutions of (10). From [3, 4, 10] we know that $\tilde{m}=m$. Also trivially $c \leqslant \tilde{c}$. Now for each $\gamma \in \Gamma$ we observe that setting $\tilde{\gamma}(t)=|\gamma(t)|$ one has

$$
\|\nabla \tilde{\gamma}(t)\|_{2}^{2} \leqslant\|\nabla \gamma(t)\|_{2}^{2} \quad \text { and } \int_{\mathbb{R}^{N}} G(\tilde{\gamma}(t)) d x=\int_{\mathbb{R}^{N}} G(\gamma(t)) d x .
$$

Thus $\tilde{\gamma} \in \tilde{\Gamma}$ and $S(\tilde{\gamma}) \leqslant S(\gamma)$. This shows that $\tilde{c} \leqslant c$ and ends the proof.

\section{ACKNOWLEDGMENTS}

The authors wish to thank Masahito Ohta and Grozdena Todorova for the interest they have taken in this work and for fruitful discussions. They are grateful to Mariana Hărăgus for fruitful discussions. They also wish to thank the referee for his careful reading and suggestions which helped to improve the paper. 


\section{REFERENCES}

[1] R. Adams, Sobolev spaces, Pure and Applied Mathematics, 65, Academic Press, (1975). MR0450957 (56:9247)

[2] H. Berestycki and T. Cazenave, Instabilité des états stationnaires dans les équations de Schrödinger et de Klein-Gordon non linéaires, C. R. Acad. Sci. Paris 293, (1981), 489-492 and Publications du Laboratoire d'Analyse Numérique, Université de Paris VI. MR646873 (84f:35120)

[3] H. Berestycki, T. Gallouet and O. Kavian, Équations de champs scalaires euclidiens non linéaires dans le plan, C. R. Acad. Sci. Paris 297, (1983), 307-310 and Publications du Laboratoire d'Analyse Numérique, Université Pierre et Marie Curie. MR734575 (85e:35041)

[4] H. Berestycki and P.L. Lions, Nonlinear scalar field equations I, Arch. Ration. Mech. Anal., 82, (1983), 313-346. MR695535 (84h:35054a)

[5] J. Byeon, L. Jeanjean and K. Tanaka, Standing waves for nonlinear Schrödinger equations with a general nonlinearity: One and two dimensional cases, Comm. Partial Differential Equations 33, 4-6, (2008), 1113-1136. MR2424391

[6] T. Cazenave, Semilinear Schrödinger equations, Courant Lecture Notes in Mathematics, 10, (2003). MR 2002047 (2004j:35266)

[7] T. Cazenave and A. Haraux, An introduction to semilinear evolution equations, Oxford Lecture Series in Mathematics and its Applications, 13, Oxford University Press, (1998). MR:1691574 (2000e:35003)

[8] T. Cazenave and P.L. Lions, Orbital stability of standing waves for some nonlinear Schrödinger equations, Comm. Math. Phys. 85, 4, (1982), 549-561. MR677997(84i:81015)

[9] J. Chen and B. Guo, Strong instability of standing waves for a nonlocal Schrödinger equation, Phys. D 227, (2007), 142-148. MR2332502 (2008c:35301)

[10] S. Cingolani, L. Jeanjean and S. Secchi, Multi-peak solutions for magnetic NLS equations without non-degeneracy condition, to appear in ESAIM Contrôle Optim. Calc. Var. DOI: 10.1051/cocv:2008055.

[11] S. Coleman, V. Glaser and A. Martin, Action minima among solutions to a class of Euclidean scalar field equations, Comm. Math. Phys. 58, 2, (1978), 211-221. MR0468913 (57:8716)

[12] S. Ibrahim, M. Majdoub and N. Masmoudi, Global solutions for a semilinear, twodimensional Klein-Gordon equation with exponential-type nonlinearity, Comm. Pure Appl. Math. 59, 11, (2006), 1639-1658. MR2254447 (2007h:35229)

[13] L. Jeanjean and K. Tanaka, A remark on least energy solutions in $R^{N}$, Proc. Amer. Math. Soc. 131, 8, (2003), 2399-2408. MR1974637(2004c:35127)

[14] L. Jeanjean and K. Tanaka, A note on a mountain pass characterization of least energy solutions, Adv. Nonlinear Stud. 3, 4, (2003), 445-455. MR2017241 (2004i:34228)

[15] S. Le Coz, A note on Berestycki-Cazenave's classical instability result for nonlinear Schrödinger equations, Adv. Nonlinear Stud. 8, 3, (2008), 455-463. MR2426909

[16] Y. Liu, Strong instability of solitary-wave solutions to a Kadomtsev-Petviashvili equation in three dimensions, J. Differential Equations 180, 1, (2002), 153-170. MR.1890602 $(2003 \mathrm{j}: 35278)$

[17] Y. Liu, M. Ohta and G. Todorova, Strong instability of solitary waves for nonlinear KleinGordon equations and generalized Boussinesq equations, Ann. Henri Poincaré 24, 4, (2007), 539-548. MR2334991

[18] Y. Liu, X.-P. Wang and K. Wang, Instability of standing waves of the Schrödinger equation with inhomogeneous nonlinearity, Trans. Amer. Math. Soc. 358, (2006), 2105-2122. MR2197450 (2006k:35275)

[19] H. Nawa, Asymptotic profiles of blow-up solutions of the nonlinear Schrödinger equation with critical power nonlinearity, J. Math. Soc. Japan 46, 4, (1994), 557-586. MR1291107 (95g:35195)

[20] O. A. Nielsen, An introduction to integration and measure theory, Canadian Mathematical Society Series of Monographs and Advanced Texts, Wiley-Interscience, (1997). MR 1468232 (98j:28002)

[21] M. Nakamura and T. Ozawa, The Cauchy problem for nonlinear Klein-Gordon equations in the Sobolev spaces, Publ. Res. Inst. Math. Sci. 37, 3, (2001), 255-293. MR1855424 (2002k:35213) 
[22] M. Ohta and G. Todorova, Strong instability of standing waves for nonlinear Klein-Gordon equations, Discrete Contin. Dyn. Syst. 12, 2, (2005), 315-322. MR2122169 (2005k:35289)

[23] M. Ohta and G. Todorova, Strong instability of standing waves for the nonlinear KleinGordon equation and the Klein-Gordon-Zakharov system, SIAM J. Math. Anal. 38, 6, (2007), 1912-1931. MR2299435 (2008a:35198)

[24] J. Shatah, Unstable ground state of nonlinear Klein-Gordon equations, Trans. Amer. Math. Soc. 290, 2, (1985), 701-710. MR792821 (86k:35088)

[25] J. Shatah and W. Strauss, Instability of nonlinear bound states, Comm. Math. Phys. 100, 2, (1985), 173-190. MR804458 (87b:35159)

[26] W. Strauss, Existence of solitary waves in higher dimensions, Comm. Math. Phys. 55, 2, (1977), 149-162. MR0454365 (56:12616)

Laboratoire de Mathématiques, Université de Franche-Comté, 25030 Besançon Cedex, France

E-mail address: louis.jeanjean@univ-fcomte.fr

Laboratoire de Mathématiques, Université de Franche-Comté, 25030 Besançon Cedex, France

E-mail address: slecoz@univ-fcomte.fr

Current address: Department of Mathematics, Scuola Internazionale Superiore di Studi Avanzati, Via Beirut 2-4, 34014 Trieste, Italy

E-mail address: lecoz@sissa.it 